\title{
Computation of mass loss from viscous accretion disc in presence of cooling
}

\author{
Santabrata Das \\ ARCSEC, Sejong University, Seoul, South Korea \\ Indranil Chattopadhyay * \\ Department of Astronomy and Space Science, Chungnam National Univ, Daejeon, \\ South Korea
}

\begin{abstract}
Rotating accretion flow may undergo centrifugal pressure mediated shock transition even in presence of various dissipative processes, such as viscosity and cooling mechanism. The extra thermal gradient force along the vertical direction in the post shock flow drives a part of the accreting matter as bipolar outflows which are believed to be the precursor of relativistic jets. We compute mass loss rates from a viscous accretion disc in presence of synchrotron cooling in terms of the inflow parameters. We show cooling significantly affects the mass outflow rate, to the extent that, jets may be generated from flows with higher viscosity. We discuss that our formalism may be employed to explain observed jet power for a couple of black hole candidates. We also indicate that using our formalism, it is possible to connect the spectral properties of the disc with the rate of mass loss.
\end{abstract}

Key words:

hydrodynamics, black hole physics, accretion, accretion discs, ISM: jets and outflows

\section{Introduction}

In recent years, it has been established that AGNs and Microquasars suffer mass loss in the form of jets and outflows (Ferrari, 1998; Mirabel \& Rodriguez,

\footnotetext{
* Corresponding author.

Email addresses: sbdas@canopus.cnu.ac.kr (Santabrata Das), indra@canopus.cnu.ac.kr (Indranil Chattopadhyay ).
} 
1999). Generation of jets or outflows around gravitating centres with hard boundaries (e.g., neutron stars, YSOs etc.) are quite natural, however, it is altogether a different proposition to consider the same around a black hole. As black holes do not have either hard boundaries or intrinsic atmospheres, jets/outflows have to originate from the accreting matter onto black holes, though there is no consensus about the exact mechanism of jet formation. One of the motivation of studying black hole accretion is therefore to understand the primary mechanism in the accretion process which may be responsible for the generation of jets. In addition, recent observations have established that, whatever be the exact mechanism behind the formation of jets/outflows around black holes, the formation of jets is intrinsically linked with spectral states of the associated black hole candidates. In particular, Gallo et al. (2003) showed that quasi steady jets are generally ejected in the hard state, which suggests that the generation or quenching of jets do depend on various states of the accretion disc. Several theoretical attempts were made to explain the possible mechanisms of jet generation from accretion disc. $\mathrm{Xu} \&$ Chen (1997) reported the formation of outflows by considering self-similar solutions. Chakrabarti (1999); Das \& Chakrabarti (1999) estimated mass outflow rates in terms of inflow parameters from an inviscid advective disc. In particular, these authors showed that the centrifugal barrier may produce shock, and the post-shock disc can generate bipolar outflows. They also showed mass outflow rates depend on the strength of the centrifugal barrier, as well as, its thermal driving. Das et al. (2001b) extended this work to show that such outflows generated by accretion shock is compatible with the spectral state of the accretion disc. The shock induced relativistic outflows could be obtained if various acceleration mechanism, namely, first order Fermi acceleration at the shock (Le \& Becker, 2005), or radiation pressure (Chattopadhyav, 2005), are considered.

Recently, Chattopadhyay \& Das (2007) computed mass outflow rates from a viscous advective disc and showed that the mass outflow rate decreases with the increase of viscosity parameter. In realistic accretion disc, a variety of dissipative processes are expected to be present, and viscosity is just one of them. In absence of mass loss, $\mathrm{Gu} \& \mathrm{Lu}$ (2004) conjectured that cooling processes will not affect the nature of advective accretion solutions. However, Das (2007) explicitly showed that cooling processes play a crucial role in determining the flow variables as well as the shock properties. Therefore, it will be worthwhile to investigate, how cooling would affect the mass outflow rate from a viscous accretion disc. In presence of viscosity, as matter flows inward angular momentum decreases while specific energy increases. A cooling process unlike viscosity, only reduces the energy of the flow and leaves the angular momentum distribution un-affected. Thus the increase of flow energy due to viscous heating may be abated by incorporating cooling mechanism. As cooling is more efficient at the hotter and denser post-shock region (abbreviated as $\mathrm{CENBOL} \equiv$ CENtrifugal pressure supported BOundary Layer), the decrease 
of CENBOL energy will be more pronounced compared to the pre-shock energy. In reality, more energetic flows at the outer edge, which do not satisfy shock conditions in absence of cooling, may undergo shock transition in its presence. Consequently, more energetic CENBOL may be produced for flows with higher cooling efficiency, and hence there is a possibility of enhanced jet driving. In this paper, we would like to address these issues in detail.

In the next section, we present the model assumptions and the governing equations. In Section 3, we discuss the methodology of computing self-consistent inflow-outflow solutions and present the solutions. In Section 4, we apply our formalism on two black hole candidates to compute the mass outflow rate, and compare it with the observed jet power. In the last section we draw concluding remarks.

\section{Model Assumptions and Equations of motion}

In a disc-jet system, there are two separate flow geometries, namely, one for accretion flows and the other for outflows. Axis-symmetry and steady state conditions are assumed for the disc-jet system. In the present paper, we consider thin, viscous accretion flow in presence of synchrotron cooling. Jets are assumed to be tenuous. Since jets are in general collimated, they should have less angular momentum and therefore less differential rotation compared to the accretion disc. Thus, we ignore the effect of viscosity in jets. As jets are believed to originate from the inner part of the disc, which in our model is the CENBOL, the jet base must be described by identical local accretion flow variables (see section 3), i.e., the specific energy, the angular momentum etc of the CENBOL. Consequently, we neglect the torque between the disc and the jet at the jet base. It is to be remembered that, to keep the jets collimated, angular momentum will be reduced either by magnetic field (stochastic fields, considered in the paper, are not effective in doing so), or by radiation [see, (Chattopadhyay, 2005)], however these processes have not been considered here. In reality, back reactions on the disc in the form of extra torque at the jet base and/or feedback effect from failed jets are not altogether ruled out. To study these effects, one requires to undertake numerical simulation, which is beyond the scope of the present frame work. Moreover, jets are supposed to be colder than the accretion discs. Therefore, we assume jets to be adiabatic, at least up to its critical point. We use pseudo-Newtonian potential introduced by Paczyński \& Wiita (1980) to approximate the space time geometry around a non-rotating black hole.

A schematic structure of shocked advective accretion disc and the associated jet are presented in Fig. 1. Here, $x_{c o}$ and $x_{c i}$ are the outer and the inner critical points of the disc, respectively. The centrifugal pressure acts as a 'barrier' to 


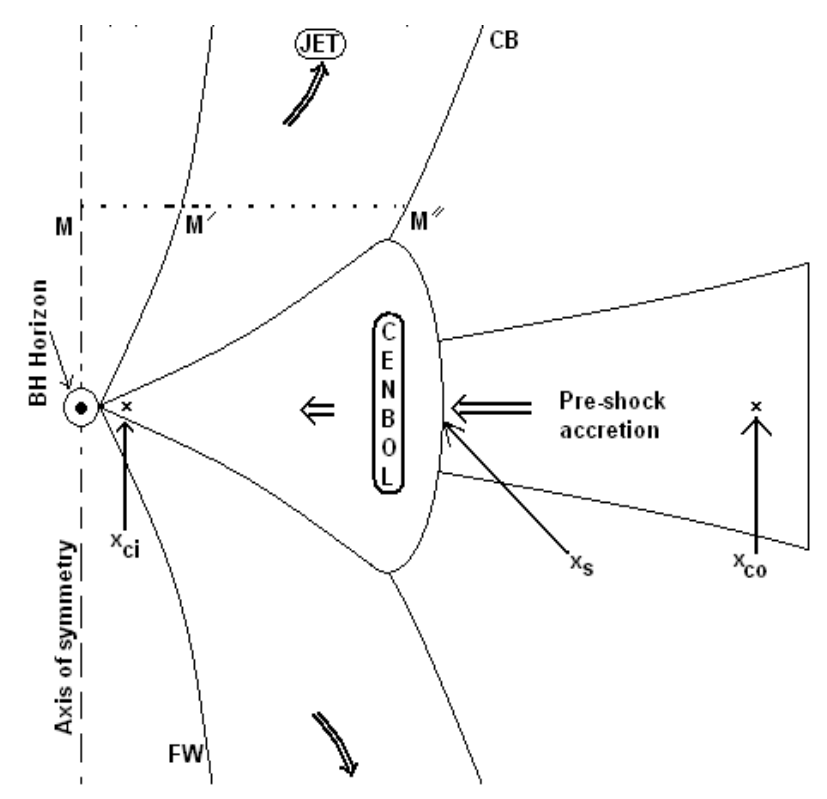

Fig. 1. A schematic diagram of disc-jet system. The outer and inner critical points $x_{c o}$ and $x_{c i}$ are marked in the figure. The shock is located at $x_{s}$. The jet geometry is bounded by $\mathrm{FW}$ and $\mathrm{CB} . \mathrm{MM}^{\prime}=x_{F W}$ and $\mathrm{MM}^{\prime \prime}=x_{C B}$ (described in the text).

the supersonic matter at $x_{c i}<x<x_{c o}$ and a shock at $x_{s}$ is formed. The postshock disc is indicated in the figure as CENBOL. At the shock, matter momentarily slows down and ultimately dives into the black hole supersonically through $x_{c i}$. Excess thermal driving in CENBOL drives a fraction of accreting matter as bipolar jet which flows within two geometric surfaces called the Funnel Wall (FW) and the Centrifugal Barrier (CB) (Molteni et al. , 1994, 1996a).

The system of units used in this paper is $2 G=M_{\mathrm{BH}}=c=1$, where $G, M_{\mathrm{BH}}$ and $c$ are the universal gravitational constant, the mass of the black hole and the speed of light, respectively. Since we use the geometrical system of units, our formalism is applicable for both the galactic and the extra galactic black hole candidates. Two separate sets of hydrodynamic equations for accretion and jet, are presented bellow.

The dimensionless hydrodynamic equations that govern the motion of accreting matter are (Chakrabarti, 1996; Das, 2007),

the radial momentum equation :

$$
u \frac{d u}{d x}+\frac{1}{\rho} \frac{d P}{d x}-\frac{\lambda^{2}(x)}{x^{3}}+\frac{1}{2(x-1)^{2}}=0,
$$

where, $u, \rho, P$, and $\lambda(x)$ are the radial flow velocity, the local density, the isotropic pressure and the local specific angular momentum, respectively. Here $x$ is the cylindrical radial coordinate. 
The baryon number conservation equation :

$$
\dot{M}=2 \pi \Sigma u x,
$$

where, $\dot{M}$ and $\Sigma$ are the mass accretion rate and the vertically integrated density, respectively. In our model, the accretion rates in the pre shock and post shock regions are different as some fraction of the accreting matter is ejected as outflow. Actually, the post-shock matter is flown into two channels - one is the accreting part (falling onto black holes through $x_{c i}$ ) and the other is the outflowing part (Molteni et al., 1994, 1996a; Chattopadhyay \& Das, 2007). More specifically, the combination of accretion and outflow rate in the post shock region remain conserved with the pre-shock accretion rate (see Eq. $3)$.

The angular momentum conservation equation :

$$
u \frac{d \lambda(x)}{d x}+\frac{1}{\Sigma x} \frac{d}{d x}\left(x^{2} W_{x \phi}\right)=0,
$$

where, $W_{x \phi}(=-\alpha \Pi)$ denotes the viscous stress, $\alpha$ is the viscosity parameter and $\Pi$ is the vertically integrated total (i.e., thermal + ram) pressure. The viscosity prescription employed in this paper was developed by Chakrabarti \& Molteni (1995) and has been employed to study advective accretion disc by a group of workers (Chakrabarti, 1996; Chakrabarti \& Das, 2004; Gu \& Lu, 2004; Das, 2007; Chattopadhyay \& Das, 2007). This viscosity prescription is more suitable for flows with significant radial velocity as it maintains angular momentum distribution continuous across the shock unlike Sakura-Sunyaev type viscosity prescription which was proposed for a Keplerian disc.

And finally, the entropy generation equation :

$$
u T \frac{d s}{d x}=Q^{+}-Q^{-},
$$

where, $s$ is the specific entropy of the flow, $T$ is the local temperature. $Q^{+}$and $Q^{-}$are the heat gained and lost by the flow, and are given by (Chakrabarti, 1996; Das, 2007; (Shapiro \& Teukolsky, 1983),

$$
Q^{+}=-\frac{\alpha}{\gamma} x\left(g a^{2}+\gamma u^{2}\right) \frac{d \Omega}{d x}
$$

and

$$
Q^{-}=\frac{\beta S_{i} a^{5}}{u x^{3 / 2}(x-1)} .
$$

Here, $g=I_{n+1} / I_{n}, n=1 /(\gamma-1), I_{n}=\left(2^{n} n !\right)^{2} /(2 n+1)$ ! (Matsumoto et al. , $1984)$, and $\gamma(=4 / 3)$ is the adiabatic index. Presently, we consider only synchrotron cooling. In the above equation, $\beta$ is the cooling parameter, and $S_{i}$ is 
the synchrotron cooling term which is independent of the flow variables and is given by,

$$
S_{i}=\frac{32 \eta \dot{m}_{i} \mu^{2} e^{4} 1.44 \times 10^{17}}{3 \sqrt{2} m_{e}^{3} \gamma^{5 / 2}} \frac{1}{2 G M_{\odot} c^{3}},
$$

where, $e$ is the electron charge, $m_{e}$ is electron mass, $\dot{m}_{i}$ is the accretion rate in units of Eddington rate, $M_{\odot}$ is solar mass, and for fully ionized plasma $\mu=0.5$. The suffix ' $i=\mp$ ' represents the quantities in the pre/post shock disc region. It is to be borne in mind that in absence of shock $\dot{m}_{+}=\dot{m}_{-}$, therefore $S_{+}=S_{-}$. Due to the uncertainties of the realistic magnetic field structure in the accretion disc, we have assumed stochastic magnetic field. The ratio between the magnetic pressure and the gas pressure is represented by $\eta$. The magnetic field strength is estimated by assuming partial equipartition $(\eta \leq 1)$ of the magnetic pressure with the gas pressure. In this paper, we have ignored bremsstrahlung cooling, since it is a very inefficient cooling process (Chattopadhyay \& Chakrabarti, 2000; Das \& Chakrabarti, 2004). The expression for bremsstrahlung cooling (Rybicki \& Lightman, 1979) in vertical equilibrium is given by,

$$
Q_{B}^{-}=\frac{B_{i}}{u x^{3 / 2}(x-1)},
$$

where

$$
B_{i}=\frac{2.016 \times 10^{-10}}{4 \pi m_{p}^{2}}\left(\frac{\mu m_{p}}{2 k_{B}}\right)^{1 / 2} \frac{\dot{m}_{i}}{2 G M_{\odot} c},
$$

where, $m_{p}$ is the proton mass and $k_{B}$ is the Boltzmann constant. For identical accretion rates

$$
\frac{S_{i}}{B_{i}}=3.26 \times 10^{7} \times \eta
$$

Therefore, it is quite evident that the synchrotron cooling is much stronger than bremsstrahlung. However, bremsstrahlung photons may interact with the accreting gas itself and in that sense bremsstrahlung may be important. Such complicated situation is not addressed in the present paper. We have also not considered inverse-Compton, since that will require a proper two temperature solution which is also beyond the scope of the present effort.

In the present paper, we have chosen $\dot{m}_{-}=0.1$ and $\eta=0.1$ as the representative case, until stated otherwise.

Under the adiabatic assumption for the jet, the momentum balance equation can be represented in the following integrated form:

$$
\mathcal{E}_{j}=\frac{1}{2} v_{j}^{2}+n a_{j}^{2}+\frac{\lambda_{j}^{2}}{2 x_{j}^{2}}-\frac{1}{2\left(r_{j}-1\right)},
$$

where, $\mathcal{E}_{j}$ and $\lambda_{j}$ are the specific energy and angular momentum of the jet, respectively. Other flow variables are the jet velocity $\left(v_{j}\right)$ and sound speed 
$\left(a_{j}\right)$. Furthermore, $x_{j}\left[=\left(x_{C B}+x_{F W}\right) / 2\right]$ and $r_{j}\left[=\left(x_{j}^{2}+y_{C B}^{2}\right)^{1 / 2}\right]$ are the cylindrical and spherical radius of the jet streamline. The functional form of the coordinates of CB and FW are [see, Chattopadhyay \& Das (2007)],

$$
\begin{gathered}
x_{C B}=\left[2 \lambda_{j}^{2} r_{C B}\left(r_{C B}-1\right)\right]^{1 / 4}, \\
x_{F W}^{2}=\lambda_{j}^{2} \frac{\left(\lambda_{j}^{2}-2\right)+\sqrt{\left(\lambda_{j}^{2}-2\right)^{2}-4\left(1-y_{C B}^{2}\right)}}{2},
\end{gathered}
$$

where, $x_{C B}$ and $x_{F W}$ are measured at the same height of jet streamline and is given by $y_{C B}=\sqrt{\left(r_{C B}^{2}-x_{C B}^{2}\right)}$.

The integrated form of mass-flux conservation equation for the jet is given by,

$$
\dot{M}_{\text {out }}=\rho_{j} v_{j} \mathcal{A}
$$

where, $\dot{M}_{\text {out }}$ is jet outflow rate and $\rho_{j}$ is the local density of the jet. The jet cross-sectional area is given by, $\mathcal{A}=2 \pi\left(x_{C B}^{2}-x_{F W}^{2}\right)$.

\section{Accretion-Ejection solution}

It is well known that matter falling onto black holes have to cross one or more critical points depending on the absence or presence of shock transition (Chakrabarti, 1996; Chakrabarti \& Das, 2004; Chattopadhyay \& Das, 2007). If the flow parameters allow shock transition then matter must cross the sonic horizon twice, once before the shock and then after the shock. The location of the latter is called the inner critical point $\left(x_{c i}\right)$ and the former is known as outer critical point $\left(x_{c o}\right)$. In absence of dissipation, the energy $(\mathcal{E})$ and angular momentum $(\lambda)$ of the flow is conserved, and therefore $x_{c i}$ and/or $x_{c o}$ are uniquely obtained in terms of $\mathcal{E}$ and $\lambda$, and consequently all possible flow solutions. $\mathcal{E}$ and $\lambda$ do not remain conserved along a dissipative flow and therefore critical points cannot be determined uniquely. To obtain solutions of a dissipative accretion flow in a simpler way, one needs to know at least one set of critical point parameters $\left(e . g_{.}, x_{c}, \lambda_{c}\right)$. Fortunately, the range of $\left(x_{c i}, \lambda_{c i}\right)$ s varies from $\left(2 r_{g} \lesssim x_{c i} \lesssim 4 r_{g}, 1.5 \lesssim \lambda_{c i} \lesssim \lambda_{m s}\right)$, where $\lambda_{c i}, \lambda_{m s}$ are the angular momentum at the inner critical point and the marginally stable orbit, respectively [e.g., Chakrabarti (1989, 1996); Chakrabarti \& Das (2004)]. Here $r_{g}$ is the Schwarzschild radius. Therefore for a viscous flow, it is easier to consider $x_{c i}$ and $\lambda_{c i}$ as parameters for solving the flow equations, along with the viscosity parameter $\alpha$ (Chakrabarti \& Das, 2004; Chattopadhyay \& Das, 2007). In presence of cooling, one should also supply the accretion rate at $x_{c i}$ in addition to $\left(x_{c i}, \lambda_{c i}, \alpha\right)$. Presently, we fix accretion rate and vary $\beta$ to study the effect of cooling. Hence the existence of $x_{c o}$ can be obtained only in presence of a shock. 
In this paper, we consider infinitesimally thin adiabatic shock, generally expressed by the continuity of energy flux, mass flux and momentum flux across the shock, and is generally called Rankine-Hugoniot ( $\mathrm{RH}$ ) shock conditions. Numerical simulations [e.g., Eggum et al. (1985); Molteni et al. (1994, 1996a)] have shown that thermally driven outflows could originate from the hot inner part of the disc. When rotating matter accretes towards the black hole, centrifugal force acts as a barrier, inducing the formation of shock. At the shock, flow temperature rises sharply as the kinetic energy of the flow is converted into the thermal energy. This excess thermal energy may drive a significant fraction of accreted material as outflows. Thus bulk properties such as excess thermal driving along $z$ direction is a legitimate process for mass ejections.

The modified Rankine-Hugoniot shock conditions in presence of mass loss are Chattopadhyay \& Das (2007), and references therein],

$$
\mathcal{E}_{+}=\mathcal{E}_{-} ; \quad \dot{M}_{+}=\dot{M}_{-}-\dot{M}_{\text {out }}=\dot{M}_{-}\left(1-R_{\dot{m}}\right) ; \quad \Pi_{+}=\Pi_{-},
$$

Assuming the jet to be launched with the same specific energy, angular momentum and density as the post-shock disc, the expression for relative mass outflow rate is given by (Chattopadhyay \& Das, 2007),

$$
R_{\dot{m}}=\dot{M}_{\text {out }} / \dot{M}_{-}=\frac{R v_{j}\left(x_{s}\right) \mathcal{A}\left(x_{s}\right)}{4 \pi \sqrt{\frac{2}{\gamma}} x_{s}^{3 / 2}\left(x_{s}-1\right) a_{+} u_{-}},
$$

where, the compression ratio is defined as $R=\Sigma_{+} / \Sigma_{-}$. Since, the information of $R_{\dot{m}}$ is in the shock condition itself, we need to solve accretion-ejection equations simultaneously. The method to do so is as follows:

(a) we assume $R_{\dot{m}}=0,\left(\dot{m}_{-}=\dot{m}_{+}\right)$, and with the supplied values of $\left(x_{c i}\right.$, $\left.\lambda_{c i}, \alpha, \beta\right)$ we integrate Eqs. (1a-d) outwards along the sub-sonic branch of the post-shock region. Equation (3) is used to compute the pre-shock flow quantities, which are employed to integrate outwards to find the location of $x_{c o}$. The location of the jump for which $x_{c o}$ exists is the virtual shock location $\left(x_{s}^{\prime}\right)$.

(b) Once $x_{s}^{\prime}$ is found out, we assign $\mathcal{E}_{j}=\mathcal{E}\left(x_{s}^{\prime}\right)$ and $\lambda_{j}=\lambda\left(x_{s}^{\prime}\right)$ to solve the jet equations and compute the corresponding $R_{\dot{m}}$.

(c) We use this value of $R_{\dot{m}}$ in Eq. (3) and again calculate the shock location. (d) When the shock locations converge we have the actual shock location $\left(x_{s}\right)$, and the corresponding $R_{\dot{m}}$ is the mass outflow rate.

In other words, we are launching jets with same $\mathcal{E}, \lambda$, and $\rho$ as that of the shock.

Presently, we consider viscosity and synchrotron cooling process as the source of dissipation in the flow. Viscosity reduces the angular momentum, while increases the energy as the flow accretes towards the central object. Cooling process on the other hand, decreases the flow energy inwards while leaving the angular momentum distribution unaffected. For proper understanding of 


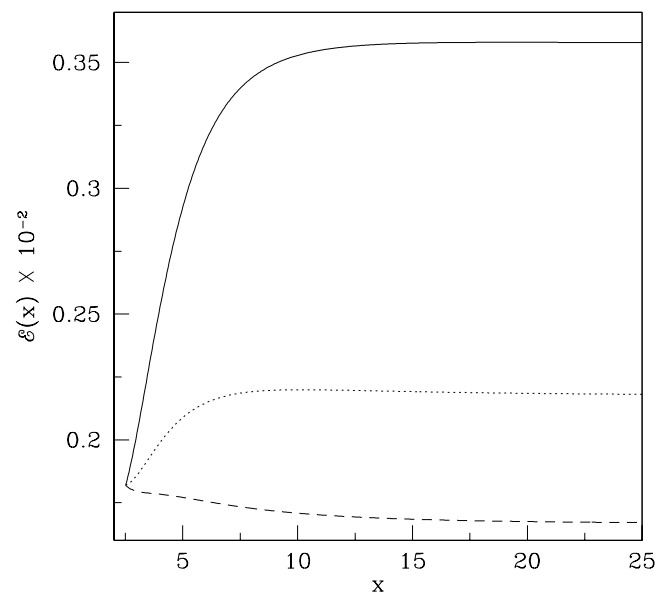

Fig. 2. $\mathcal{E}(x)$ with $x$ is plotted for $\beta=0$ (dashed), 0.01 (dotted) and 0.036 (solid). Other parameters are $\left(\mathcal{E}_{c i}, \lambda_{c i}\right)=(0.00182,1.73)$ and $\alpha=0.001$.

the effect of viscosity and cooling on determining mass outflow rates we need to fix $(\mathcal{E}, \lambda)$ at some length-scale (around inner or outer boundary), and then vary $\alpha$ and $\beta$.

As $x_{c i}$ is very close to the horizon, fixing $\left(\mathcal{E}_{c i}, \lambda_{c i}\right)$ at $x_{c i}$ is almost equivalent to fixing the inner boundary flow quantities. In Fig. 2 , we plot $\mathcal{E}(x)$ with $x$ for $\beta=0$ (dashed), 0.001 (dotted) and 0.0036 (solid), where the inner boundary flow quantities are $\left(\mathcal{E}_{c i}, \lambda_{c i}\right)=(0.00182,1.73)$ and $\alpha=0.001$. For the cooling free solution (dashed), the energy of the flow increases inwards due to viscosity. For solutions with significant cooling (dotted, solid), the increase in energy due to viscous heating is completely over shadowed, causing the energy to decrease towards the black hole. Increase in cooling efficiency signifies, matter with higher energies at the outer boundary, falls into the black hole with identical $\mathcal{E}_{c i}$. If standing shocks form, then under these circumstances energy at the shock will increase with $\beta$. In the following, we discuss the role of viscous heating and synchrotron cooling in determining the mass outflow rate.

In Fig. 3, we present a global inflow-outflow solution. In the top panel, the Mach number $M$ of the accretion flow is plotted with $\log (x)$. The solid curve represents shock induced accretion solution. The inflow parameters are $x_{c i}=$ 2.444, $\lambda_{i}=1.75, \alpha=0.005$, and $\beta=0.01$ (for these parameters $\mathcal{E}_{c i}=0.0018$ ). In the lower panel, the outflow Mach number $M_{j}$ is plotted with $\log \left(x_{j}\right)$. In presence of mass loss, the shock forms at $x_{s}=21.64$ denoted by the vertical line in the top panel, and the outflow is launched with energy and angular momentum at the shock $\left(\mathcal{E}_{s}, \lambda_{s}=0.00175,1.766\right)$. The outflow is plotted up to its sonic point $\left(x_{j c}=68.83\right)$, and the corresponding relative mass outflow rate is $R_{\dot{m}}=0.0816$. 

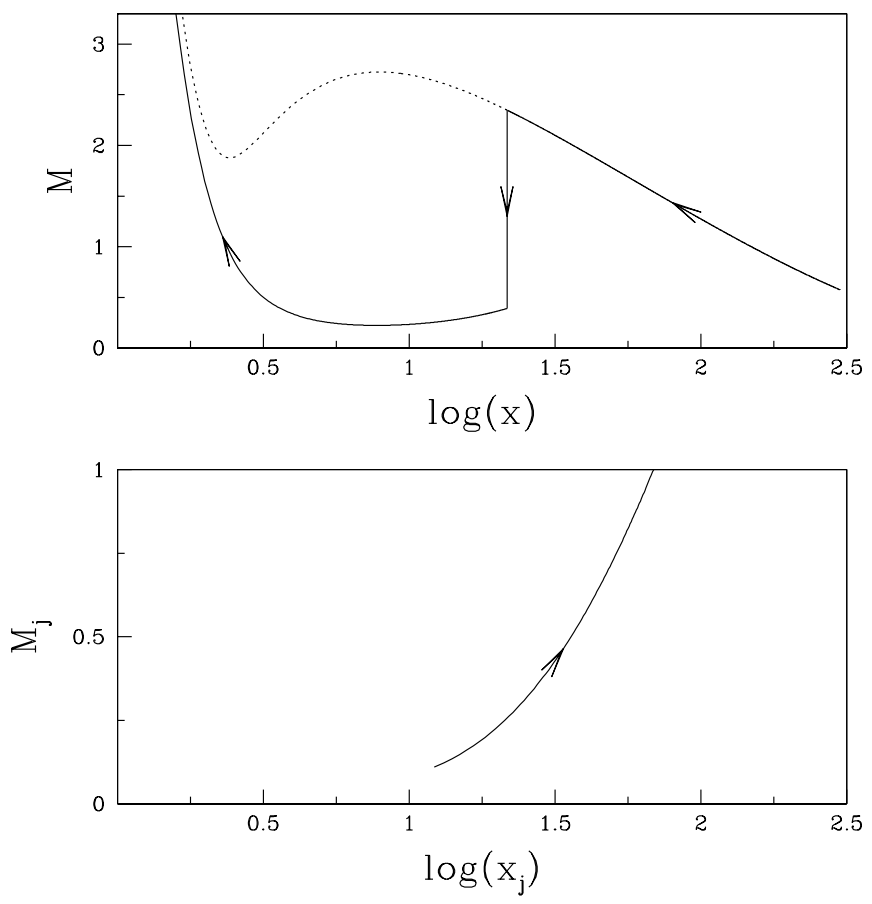

Fig. 3. Upper panel: Inflow Mach number $(M=u / a)$ with $\log (x)$. The inflow parameters are $x_{c i}=2.444, \lambda_{i}=1.75, \alpha=0.005$, and $\beta=0.01$ where, $x_{s}=21.64$, $\mathcal{E}_{s}=0.00175, \lambda_{s}=1.766 x_{c o}=166.57, \lambda_{o}=1.799$. The dotted curve is the shock free solution. Lower panel: Outflow Mach number $\left(M_{j}=v_{j} / a_{j}\right)$ with $\log \left(x_{j}\right)$, the outflow critical point $x_{j c}=68.63\left(r_{j c}=270.8\right)$, and the jet coordinates at the base is given by $x_{j b}=12.2\left(r_{j b}=21.24\right)$. The relative mass loss rate is $R_{\dot{m}}=0.0816$.

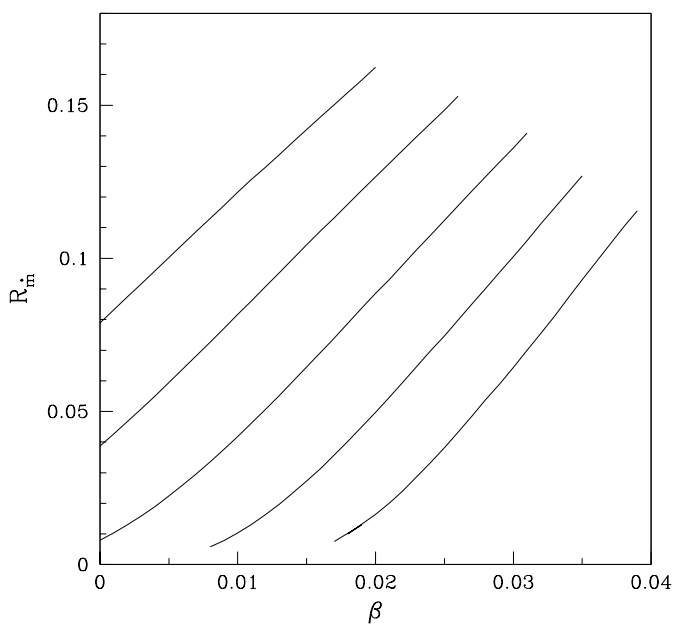

Fig. 4. Variation of $R_{\dot{m}}$ with $\beta$ for $\alpha=0-0.02$ (left to right with $d \alpha=0.005$ ). $\mathcal{E}_{c i}=0.0018$ and $\lambda_{c i}=1.75$. 


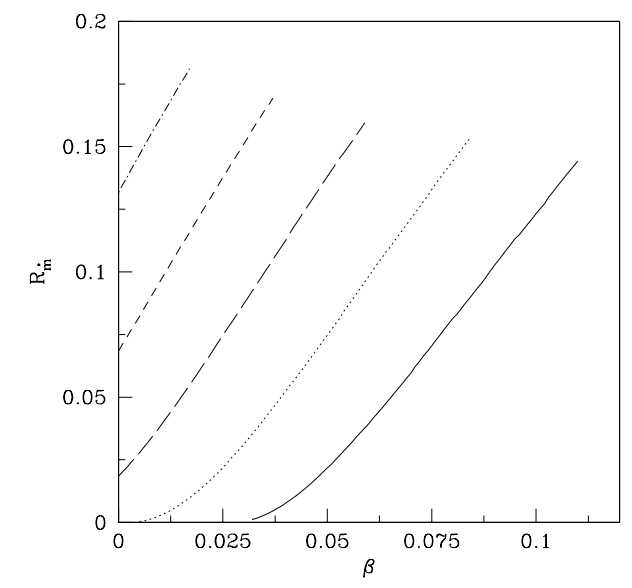

Fig. 5. $R_{\dot{m}}$ is plotted with $\beta$ for $\mathcal{E}_{c i}=-0.001 \rightarrow 0.003$ (right to left, $d \mathcal{E}_{c i}=0.001$ ). Other parameters are $\lambda_{c i}=1.73$ and $\alpha=0.001$.

To present the global solution, Fig. 3 was obtained only for a set of input parameters, namely $\left(\mathcal{E}_{c i}, \lambda_{c i}, \alpha, \beta\right)$. We would now proceed to find the explicit dependence of $R_{\dot{m}}$ on these parameters. In Fig. 4, we plot the mass outflow rates $\left(R_{\dot{m}}\right)$ with the cooling parameter $\beta$, for $\alpha=0-0.02$ (left to right for $d \alpha=0.005)$. All the curves are drawn for $\mathcal{E}_{c i}=0.0018$ and $\lambda_{c i}=1.75$. Figure 4 confirms our earlier investigation that $R_{\dot{m}}$ decreases with increasing viscosity parameter (Chattopadhyay \& Das, 2007). However, it may be noticed that for fixed $\alpha, R_{\dot{m}}$ increases with $\beta$. For a given $\alpha$, the energy at the shock increases with $\beta$ (e.g., Fig. 2), and since the post-shock region (i.e., CENBOL) is the base of the jet, the jets are launched with higher driving force. This causes $R_{\dot{m}}$ to increase with $\beta$. It is to be noted, the two extreme curves (i.e., for $\alpha=0.015,0.02)$ on the right show that, for $\beta=0$ there is no outflow, but in presence of sufficient cooling steady jets reappear. As $\alpha$ is increased, $R_{\dot{m}}$ decreases due to the gradual reduction of sufficient driving at the jet base, and beyond a critical $\alpha$ (say, $\alpha_{\text {cri }}$ ) outflow rate vanishes (Chattopadhyay \& Das, 2007). For flows with $\alpha>\alpha_{\text {cri }}$, the required jet driving could be generated by considering sufficiently high $\beta$. In other words, to get steady outflows in the realm $\alpha>\alpha_{\text {cri }}$, there is a non-zero minimum value of $\beta$ (say, $\beta_{m}$ ) corresponding to each $\alpha$. Furthermore, for each $\alpha$ there is a cut-off in $R_{\dot{m}}$ at the higher end of $\beta$ (say, $\beta_{\text {cri }}$ ), since standing shock conditions are not satisfied there. Nonsteady shocks may still form in those regions, and the investigation of such phenomena will be reported elsewhere.

In Fig. 5, $R_{\dot{m}}$ is plotted with $\beta$ for $\mathcal{E}_{c i}=-0.001$ (solid), 0.0 (dotted), 0.001 (big dashed), 0.002 (small dashed) and 0.003 (dash-dotted). Other parameters are $\lambda_{c i}=1.73$ and $\alpha=0.001$. For a given $\beta$, mass outflow rate increases with $\mathcal{E}_{c i}$. Higher $\mathcal{E}_{c i}$ corresponds to more energetic flow, and if these flows produce shock, we get higher $R_{\dot{m}}$. On the other hand, even for same $\mathcal{E}_{c i}$, higher shock 

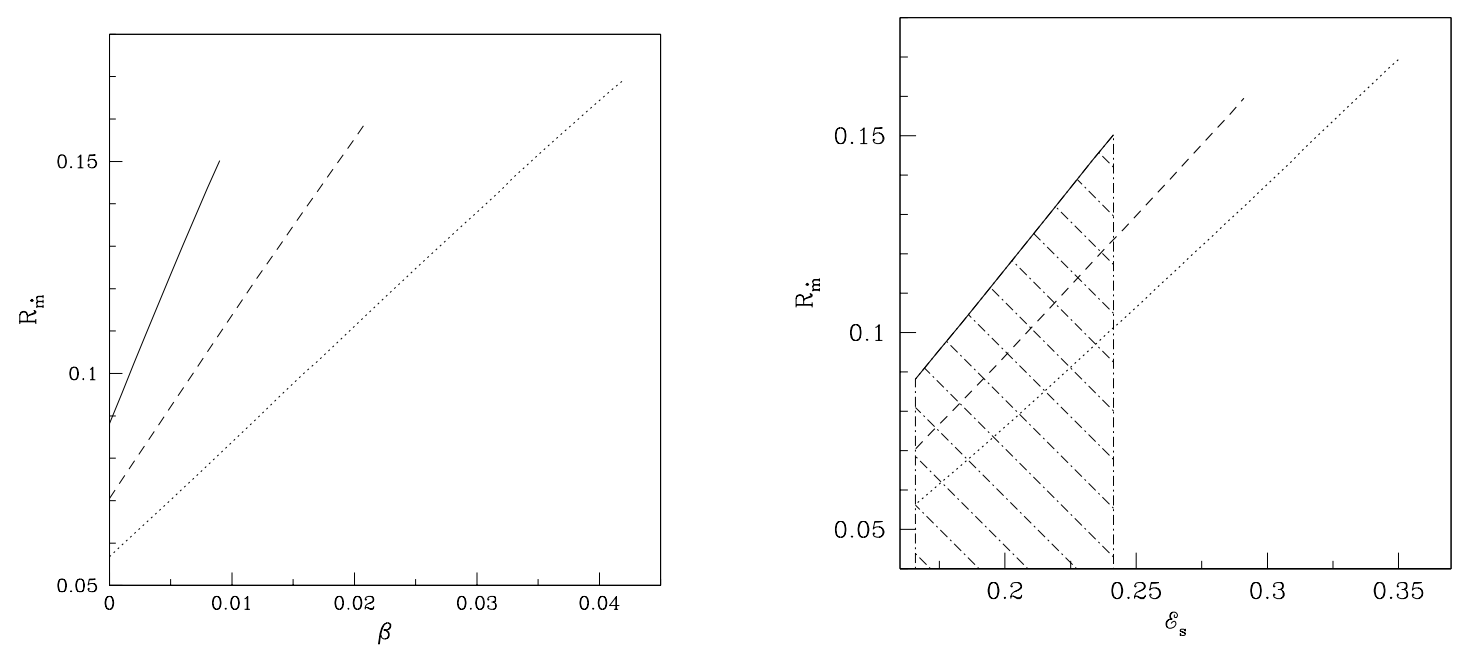

Fig. 6. (a) Variation of $R_{\dot{m}}$ with $\beta$ for $\lambda_{c i}=1.73$ (dotted) 1.75 (dashed) and 1.77 (solid). $\mathcal{E}_{c i}=0.0018$ and $\alpha=0.001$. (b) Variation of $R_{\dot{m}}$ with $\mathcal{E}_{s}$, for parameters same as Fig. 6a.

energy is ensured with the increase of $\beta$, and consequently higher $R_{\dot{m}}$ are produced. The solutions corresponding to $\mathcal{E}_{c i}=0$ (dotted) and $\mathcal{E}_{c i}=-0.001$ (solid) show that $R_{\dot{m}} \rightarrow 0$ as $\beta \rightarrow 0$. In other words, in presence of cooling, flows with bound energies at $x_{c i}$ may also produce outflows. Thus it is clear that shock energy plays an important role in determining the rate of mass loss from the disc. Previous studies of computation of mass outflow rates from inviscid and viscous disc showed that the angular momentum at the shock dictates the mass outflow rates, because higher angular momentum produces higher centrifugal driving for the jet. This lead us to investigate the role of angular momentum of the disc in determining the mass outflow rates, when cooling is present.

In Fig. 6a, $R_{\dot{m}}$ is plotted with $\beta$ for $\lambda_{c i}=1.73$ (dotted), 1.75 (dashed) and 1.77 (solid), where $\mathcal{E}_{c i}=0.00182$, and $\alpha=0.001$ are kept fixed for all the curves. For negligible cooling $(\beta \sim 0)$, higher angular momentum flow generates higher $R_{\dot{m}}$. As the centrifugal pressure produces the shock, which in turn drives the jet, it is not surprising that flows with larger angular momentum will produce higher $R_{\dot{m}}$. Similar trend is maintained for nonzero $\beta$. For a given $\lambda_{c i}$, the energy at the shock $\left(\mathcal{E}_{s}\right)$ increases with $\beta$. Thus the combined effects of centrifugal and thermal driving increase the mass outflow rate. We do see that there is a cut-off in $R_{\dot{m}}$ corresponding to each angular momentum at $\beta \geq \beta_{\text {cri }}$. For lower angular momentum flow $\beta_{\text {cri }}$ is higher. To illustrate the effects of thermal driving and centrifugal driving of the jet, in Fig. 6b, we have plotted $R_{\dot{m}}$ with $\mathcal{E}_{s}$ for $\lambda_{c i}=1.73$ (dotted), 1.75 (dashed) and 1.77 (solid), for the same 


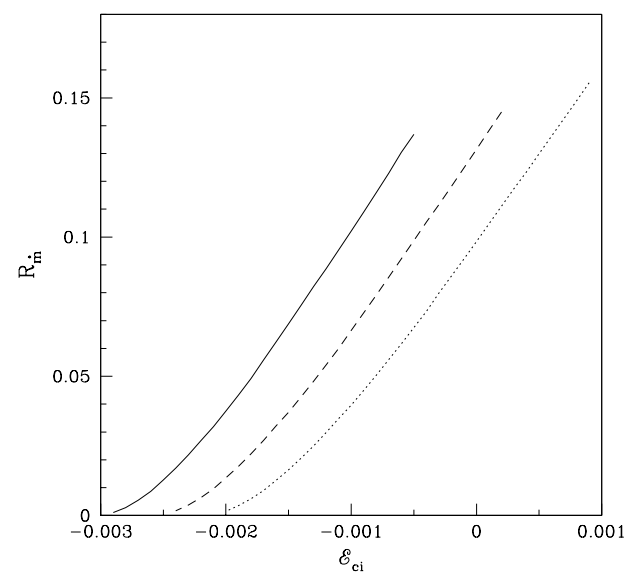

Fig. 7. $R_{\dot{m}}$ is plotted with $\mathcal{E}_{c i}$ for $\lambda_{c i}=1.73$ (dotted), $\lambda_{c i}=1.74$ (dashed), $\lambda_{c i}=1.75$ (solid). Other parameters are $\alpha=0.001$, and $\beta=0.06$.

set of $\mathcal{E}_{c i}$ and $\alpha$ as in the previous figure. It is to be remembered that $\mathcal{E}_{s}$ is not a new parameter but is calculated at the shock for the same range of $\beta$ variation as in Fig. 6a. In the shaded region, $R_{\dot{m}}$ is higher for higher $\lambda_{c i}$. As long as the shock energy is similar, higher angular momentum results in greater centrifugal driving for the outflowing matter. However, lower angular momentum flow can sustain higher energies across the shock [e.g., Fig. 3 of Das et al. (2001a)]. For high enough $\mathcal{E}_{s}$, the thermal driving starts to dominate over the centrifugal pressure, and results in higher $R_{\dot{m}}$ even for lower angular momentum flow.

In Fig. $7, R_{\dot{m}}$ is plotted as a function of $\mathcal{E}_{c i}$, for various values of $\lambda_{c i}=1.73$ (dotted), $\lambda_{c i}=1.74$ (dashed), $\lambda_{c i}=1.75$ (solid). The other flow parameters are $\alpha=0.001$ and $\beta=0.06$. This figure distinctly shows that even if the accreting flow starts with unbound energy and produces shock induced outflow, significant cooling closer to the black hole turns the unbound energy to bound energy.

\section{Astrophysical application}

In our solution procedure, we have employed three different constant parameters $\beta, \eta$ and $\dot{m}$ to determine the cooling process. A cooling mechanism might depend on various other physical processes apart from its usual dependence on the flow variables. In general, $\dot{m}$ regulates cooling, however to obtain a cooling free solution one needs to consider $\dot{m}=0$, which is meaningless. We have simplified all such complications by introducing $\beta$ as a control-parameter for cooling. A simple inspection of Eq. (1d), shows that for a given set of $(u, a, x)$, identical cooling rates may be obtained by rearranging the values of $\beta, \eta$ and 
$\dot{m}$. It must be noted that, introduction of $\beta$ and $\eta$ do not increase the parameters of our solution, instead these are used to control the cooling efficiency and the magnetic field strength, about which there is no prior knowledge. In the previous section, we have fixed the values of $\dot{m}_{-}$and $\eta$, and controlled the cooling term by $\beta$.In this section, we have fixed the value of $\beta$ to unity, and allowed physical parameters, such as $\dot{m}_{+}$and $\eta$ to dictate the cooling term.

It is a matter of interest to estimate how much matter, energy and angular momentum enter into the black hole. In the present paper, the amount of mass fed to the disc is given by $\dot{m}_{-}$. The rate at which matter is being accreted into the black hole and the rate of mass loss are self-consistently computed as $\dot{m}_{+}$ and $\left(\dot{m}_{-}-\dot{m}_{+}\right)$. It has been shown in Chattopadhyay \& Das (2007) that the specific angular momentum of the flow close to the horizon, is almost same as $\lambda_{c i}$. The actual value of $\mathcal{E}$ close to the black hole should be slightly higher than $\mathcal{E}_{c i}$. One has to quote the actual value of $\mathcal{E}$ close to the horizon. However, these numbers are obtained using pseudo-Newtonian potential and may not be consistent as general relativistic effects are important at such distances.

We have applied our formalism to calculate the mass outflow rates from two black hole candidates M87 and Sgr A*. M87 is supposed to harbour a super massive black hole $\left[M_{\mathrm{BH}}=3 \times 10^{9} M_{\odot}\right.$ (Ford et al., 1994$\left.)\right]$. The estimated accretion rate is $\dot{M}_{-} \sim 0.13 M_{\odot} \mathrm{yr}^{-1}$ (Reynolds et al. , 1996). The mass of the central black hole and the accretion rate of Sgr $\mathrm{A}^{*}$ are $M_{\mathrm{BH}}=2.6 \times 10^{6} M_{\odot}$ (Schodel et al. , 2002) and $\dot{M}_{-} \sim 8.8 \times 10^{-7} M_{\odot} \mathrm{yr}^{-1}$ (Yuan et al. , 2002). The accretion disc around the black hole in Sgr $\mathrm{A}^{*}$ is supposed to be radiatively in-efficient and of higher viscosity (Falcke, 1999). For both the cases we have set $\beta=1$, so the cooling mechanism is purely dictated by $\dot{m}$ and $\eta$. To simplify further, we have chosen $\eta=0.01$ for both the objects. The accretion rates (in terms of Eddington rate) for M87 is given by $\dot{m}_{-}=1.89 \times 10^{-2}$ and that for Sgr $\mathrm{A}^{*}$ is $\dot{m}_{-}=1.47 \times 10^{-4}$, therefore Sgr $\mathrm{A}^{*}$ is dimmer than M87. With proper choice of $\alpha$ and $x_{c i}$, and $\lambda_{c i}$ (see Table 1), we compute $R_{\dot{m}}$ (consequently $\dot{m}_{+}$) for both the objects mentioned above. The typical size of such a subKeplerian disc should be around a thousand Schwarzschild radii across the central object. Accordingly we have set the outer boundary at $X_{T}=500 r_{g}$, and have provided the typical value of angular momentum at such distance $\left(\lambda_{T}\right)$ for both the objects. For M87, the computed values of mass outflow rate and shock location are $R_{\dot{m}}=0.073$ and $x_{s}=40.57$. In case of Sgr $\mathrm{A}^{*}$, the estimated values of mass outflow rate and shock location are $R_{\dot{m}}=0.1049$ and $x_{s}=14.415$.

Assuming the jet's luminosity is significant only at the lobes (where, the jet energy is mostly dissipated), the maximum luminosities of M87 and Sgr A* jets, estimated from the computed values of respective $R_{\dot{m}}$, are given in Table 1. Considering $10 \%$ radiative efficiency at the jet lobe the jet-luminosities for both M87 and Sgr A*, agree well with the observed values (Revnolds et al., 
Table 1: Predicted values of $R_{\dot{m}}$ and jet power for M87 and Sgr A*.

\begin{tabular}{|c|c|c|c|c|c|c|c|c|c|c|}
\hline Object & $\begin{array}{c}M_{\mathrm{BH}} \\
M_{\odot}\end{array}$ & $\begin{array}{c}\dot{M}_{-} \\
M_{\odot} / \mathrm{yr}\end{array}$ & $\alpha$ & $\begin{array}{c}x_{c i} \\
r_{g}\end{array}$ & $\begin{array}{c}\lambda_{c i} \\
r_{g}\end{array}$ & $\begin{array}{c}\dot{m}_{+} \\
\dot{M}_{E d d}\end{array}$ & $\begin{array}{c}x_{s} \\
r_{g}\end{array}$ & $\begin{array}{c}\lambda_{T} \\
c r_{g}\end{array}$ & $\begin{array}{c}R_{\dot{m}} \\
\%\end{array}$ & $\begin{array}{c}L_{j e t}^{\max } \\
\mathrm{erg} / \mathrm{s}\end{array}$ \\
\hline M87 & $\begin{array}{c}3.0 \\
\times 10^{9}\end{array}$ & 0.13 & 0.010 & 2.367 & 1.78 & $\begin{array}{c}1.75 \\
\times 10^{-2}\end{array}$ & 40.57 & 2.01 & 7.3 & $\begin{array}{c}5.36 \\
\times 10^{44}\end{array}$ \\
\hline Sgr A* & $\begin{array}{c}2.6 \\
\times 10^{6}\end{array}$ & $\begin{array}{c}8.80 \\
\times 10^{-7}\end{array}$ & 0.015 & 2.548 & 1.71 & $\begin{array}{c}1.32 \\
\times 10^{-4}\end{array}$ & 14.42 & 2.44 & 10.5 & $\begin{array}{c}5.2 \\
\times 10^{39}\end{array}$ \\
\hline
\end{tabular}

1996; Falcke \& Biermann, 1999). Moreover, the size of the computed jet base for M87 is $\sim 2 x_{s} \sim 80 r_{g}$. Junor et al. (1999) and Biretta et al. (2002) have estimated the base of jet to be less than $100 r_{g}$ from the central black hole, and probably greater than $30 r_{g}$. Evidently our estimate of the jet base agrees quite well with the observations. There is no stringent upper limit of the jet base for Sgr $\mathrm{A}^{*}$, however, our computation gives a result which is acceptable in the literature (Falcke, 1999). We have also provided an estimate of angular momentum at $X_{T}$. For Sgr $\mathrm{A}^{*}$, our estimated $\lambda_{T}$ is comparable with the result of Coker \& Melia (1997). However, no reliable estimate of $\lambda_{T}$ for M87 is currently available. In terms of physical units, various flow variables for M87 are given by, $\dot{M}_{\text {out }} \sim 0.009 M_{\odot} \mathrm{yr}^{-1}, \dot{M}_{+} \sim 0.119 M_{\odot} \mathrm{yr}^{-1}, \mathcal{E}_{c i}=3.1 \times 10^{17} \mathrm{erg} \mathrm{g}^{-1}$, $x_{s} \sim 3.61 \times 10^{16} \mathrm{~cm}, \lambda_{c i} \sim 4.75 \times 10^{25} \mathrm{~cm}^{2} \mathrm{~s}^{-1}$, and $\lambda_{T} \sim 5.36 \times 10^{25} \mathrm{~cm}^{2} \mathrm{~s}^{-1}$. Similarly for Sgr A* $\dot{M}_{\text {out }} \sim 9.1 \times 10^{-8} M_{\odot} \mathrm{yr}^{-1}, \dot{M}_{+} \sim 7.77 \times 10^{-7} M_{\odot} \mathrm{yr}^{-1}, \mathcal{E}_{c i}=4 \times 10^{18} \mathrm{erg}$ $\mathrm{g}^{-1}, x_{s} \sim 1.11 \times 10^{13} \mathrm{~cm}, \lambda_{c i} \sim 3.96 \times 10^{22} \mathrm{~cm}^{2} \mathrm{~s}^{-1}$, and $\lambda_{T} \sim 5.64 \times 10^{22} \mathrm{~cm}^{2} \mathrm{~s}^{-1}$.

In this paper, only sub-Keplerian matter distribution is chosen for the accretion disc. However, Chakrabarti \& Titarchuk (1995) and Chakrabarti \& Mandal (2006) have shown that if a mixture of Keplerian and sub-Keplerian matter is chosen, then the spectral properties of the disc is better understood. These assertions have been ratified for several black hole candidates (Smith et al. 2001, 2002). Since matter close to the black hole must be sub-Keplerian, therefore regardless of their origin, Keplerian and sub-Keplerian matter mixes to produce sub-Keplerian flow before falling onto the black hole. Such transition from two component to single component flow has been shown by various authors [e.g., Fig. 4b, of Das et al. (2001b)]. The region where such transition occurs may be called 'transition radius' $\left(X_{T}\right)$. It must be noted that, $X_{T}$ is treated as the 'outer edge' of the disc in our formalism described so far. The energy $\left(\mathcal{E}_{T}\right)$ and angular momentum $\left(\lambda_{T}\right)$ at $X_{T}$ can then easily be expressed in terms of the accretion rate of the Keplerian component $\left(\dot{M}_{K}\right)$ and the subKeplerian component $\left(\dot{M}_{S K}\right)$ (Das et al. , 2001b). Once $X_{T}, \mathcal{E}_{T}, \lambda_{T}$ is known and the net accretion rate being $\dot{M}=\dot{M}_{S K}+\dot{M}_{K}$, it is easy to calculate $R_{\dot{m}}$ following our formalism. Thus, it is possible to predict $R_{\dot{m}}$ from the spectrum of the accretion disc, if formalism of Chakrabarti \& Titarchuk (1995) is applied on our solutions. 


\section{Concluding Remarks}

The main goal of this paper was to study how dissipative processes affect the jet generation in an advective disc model. Chattopadhyay \& Das (2007) have shown that mass outflow rates decrease with increasing viscosity parameter. In the present paper, we have investigated how the mass outflow rate responds to the synchrotron cooling. The general method of the solution (succinctly described in Section 3.) is to supply $x_{c i}, \lambda_{c i}, \alpha, \beta$ and then integrate outwards to find the shock location (and consequently the mass outflow rate). Needless to say, once the above four parameters are fixed, the solution determines flow with unique outer boundary (i.e., at $X_{T}$ ). Of the four parameters, if $\alpha$ is increased, the solution corresponds to flow with higher angular momentum and lower energy at the outer boundary. On the contrary, when $\beta$ is increased then the solution corresponds to higher energy but identical angular momentum flow at the outer boundary. Consequently, more energetic flows are allowed to pass through standing shock for higher $\beta$, and hence stronger jets are produced. We have also shown that, if cooling efficiency is increased, then it is possible to produce jets even for those $\alpha$-s for which $R_{\dot{m}}$ is zero (e.g., Fig. 3). Furthermore, it has been shown that the jets are primarily centrifugal pressure driven even in presence of cooling. We notice that standing shocks in higher angular momentum flow do not exist for higher cooling efficiency, therefore steady jets are not produced. However, for higher $\beta$, low angular momentum flow can generate high enough relative mass outflow rates.

We have applied our formalism on a couple of black hole candidates, namely, Sgr $A^{*}$ and M87. Using the available accretion parameters of the above two objects as inputs, we have shown that one can predict observational estimates of jet power. Moreover, the typical size of the jet base $\left(\sim 2 x_{s}\right)$ also agrees well with observations. Le \& Becker (2005) had dealt with these two particular objects, with their methodology which also involve shocked accretion disc. The methodologies of the present paper and the work of Le \& Becker (2005) is quite different in the sense that, Le \& Becker (2005) dealt with isothermal shock while our model is based on the adiabatic shock scenario. In Le \& Becker (2005), the focus was on calculating the number densities and energy densities around an isothermal shock of an hot tenuous adiabatic rotating flow, by first order Fermi acceleration process. The energy lost at the isothermal shock, drives a small fraction of in falling gas to relativistic energies. With the given observational estimates of black hole mass, accretion rate etc of M87 and Sgr $\mathrm{A}^{*}$, they estimated the Lorentz factors of the jet. We on the other hand, have computed the thermally driven outflows from the post-shock disc, where the jets are launched with the local values (specific energy, angular momentum and density) of the disc fluid at the shock. With input values of black hole mass, accretion rate, and proper choice of viscosity parameter, inner sonic point etc we predict the shock location, the mass outflow rate. We check whether the 
predicted values are within the accepted limits or not. We do not estimate the terminal bulk Lorentz factor, since we believe one has to recast the whole framework into the relativistic domain as well as employ other accelerating processes (e.g., magnetic fields etc). One may wonder at the veracity of the two different processes employed to explain the observational estimates of jet quantities of M87 and Sgr A*, in other words, whether the jets are generated by post-shock thermal driving (we have not investigated magneto-thermal driving since this is only hydrodynamic investigation), or the jets are launched by particle acceleration processes. If one can observationally estimate the rate at which mass being ejected from the accretion disc, probably then one can ascertain the dominant effect behind jet generation. If it can be established that indeed the rate of mass loss is negligible compared to the accretion rate then probably the formalism of Le \& Becker (2005) is the more realistic jet generation mechanism. However, suffice is to say, various numerical simulation results do show (for non-dissipative as well as dissipative flows) that post-shock flow thermally drive bipolar outflows, and our effort has been to investigate how dissipative processes affect the relative mass outflow rates.

In this paper we have only discussed formation of steady jets, since we have considered only stationary shocks. Molteni et al. (1996b) have shown that, the periodic breathing of the CENBOL starts when the post shock in-fall timescale matches with the Bremsstrahlung cooling timescale. Presently, we have considered dissipative processes which are more effective in determining shock properties compared to Bremsstrahlung. Therefore, the dissipative processes considered in this paper, may trigger comparable or different shockinstabilities in the disc than that has been reported earlier (Molteni et al. 1996b). Since, the jet formation is primarily controlled by the properties of the shock, any non-steady behaviour of the shock will leave its signature on the jet. In particular, a significant oscillation of the shock (both in terms of the oscillation frequency and its amplitude) may produce periodic ejections. We are studying dynamical behaviour of the shock in presence of viscosity and synchrotron cooling using fully time dependent simulation and results will be reported elsewhere.

\section{Acknowledgements}

SD was supported by KOSEF through Astrophysical Research Center for the Structure and Evolution of the Cosmos (ARCSEC), and IC was supported by the KOSEF grant R01-2004-000-10005-0. The authors thank U. Mukherjee for suggesting improvements in the manuscript. 


\section{References}

Biretta, J. A., Junor, W., Livio, M., 2002, NewA Rev 46, 239.

Chakrabarti, S. K. 1989, ApJ 347, 365 (C89)

Chakrabarti, S. K. 1996, ApJ 464, 664.

Chakrabarti, S. K., Titarchuk, L., 1995, ApJ 455, 623.

Chakrabarti, S. K., Molteni, D., 1995, MNRAS, 417, 672.

Chakrabarti, S. K., 1999, A\&A 351, 185.

Chakrabarti, S. K., Das, S., 2004, MNRAS, 349, 649.

Chakrabarti, S. K., Mandal, S., 2006, ApJ, 642, L49.

Chattopadhyay, I., Chakrabarti, S. K., 2000, Int. Journ. Mod. Phys. D 9, 717.

Chattopadhyay, I., 2005, MNRAS, 356, 145.

Chattopadhyay, I., Das, S., 2007, New A 12, 454.

(astro-ph/0610650)

Coker, R., F., Melia, F., 1997, ApJ 488, L149.

Das, T. K., Chakrabarti, S. K., 1999, Class. Quant. Grav. 16, 3879.

Das, S., Chattopadhyay, I., Chakrabarti, S. K., 2001a, ApJ 557, 983.

Das, S., Chattopadhyay, I., Nandi, A., Chakrabarti, S. K., 2001b, A\&A 379, 683

Das, S., Chakrabarti, S. K., 2004, Int. Journ. Mod. Phys. D 13, 1955.

Das, S., 2007, MNRAS 376, 1659.

Eggum, G. E., Coroniti, F. V., Katz, J. I., 1985, ApJ, 298, L41.

Falcke, H., 1999, ASPC 186, 148.

Falcke, H., Biermann, P. L., 1999, A\&A, 342, 49.

Ferrari, A., 1998, ARA\&A 36, 539.

Ford, H. C., et al. 1994, ApJ, 435, L27.

Gallo, E., Fender, R. P., Pooley, G. G. 2003, MNRAS, 344, 60

Gu, W. M., Lu, J. F., 2004, CHIN. PHYS. LETT, 21, 2551.

Junor, W., Biretta, J. A., Livio, M., 1999, Nature 401, 891.

Le, T., Becker, P. A., 2005, ApJ 632, 476

Matsumoto, R, Kato, S., Fukue, J., Okazaki A. T., 1984, PASJ, 36, 71.

Mirabel, I. F., Rodriguez, L. F., 1999, ARA\&A 37, 409.

Molteni, D., Lanzafame, G., Chakrabarti, S. K., 1994, ApJ, 425, 161.

Molteni, D., Ryu, D., Chakrabarti, S. K., 1996a, ApJ, 470, 460.

Molteni, D., Sponholtz, H, Chakrabarti, S. K., 1996b, ApJ, 457, 805.

Paczyński, B., Wiita, P., 1980, A\&A 88, 23

Reynolds, C. S., Matteo, T. D., Fabian, A. C., Hwang, U., Canizares, C. R., 1996, MNRAS 283, L111.

Rybicki, G. B., Lightman, A. P., 1979, Radiative Processes in Astrophysics, A Wiley-InterScience Publication, New York.

Shapiro, S. L., Teukolsky, S. A., 1983, Black Holes, White Dwarfs and Neutron Stars; The Physics of Compact Object, A Wiley-InterScience Publication, New York.

Schodel, R., 2002, Nature 419, 694.

Smith, D. M., Heindl, W. A., Markwardt, C. B., Swank, J. H., 2001, ApJ 554, 
L41

Smith, D. M., Heindl, W. A., Swank, J. H., 2002, ApJ 569, 362

Xu, G., Chen, X., 1997, ApJ, 489, L29.

Yuan, F., Markoff, S., Falcke, H., 2002, A\&A 383, 854. 